\title{
Monte Carlo simulation of lung counting efficiency using a whole-body counter at a nuclear power plant
}

\author{
L. Dongming ${ }^{1,2,3 \star}$, J. Shuhai ${ }^{3}$ and L. Houwen ${ }^{4}$ \\ 1 School of Physics and Information Technology, Shaanxi Normal University, Xi' an 710119, P.R. China. \\ 2 School of Science, Xi' an Shiyou University, No.18, the Second Dianzi Road, Xi' an 710065, P.R. China. \\ 3 Xi' an Jiaotong University, No.28, Xianning Road west, Xi' an 710049, P.R. China. \\ 4 The Third Qinshan Nuclear Power Co. Ltd., Haiyan 314300, P.R. China.
}

Received 21 April 2015 - Accepted 27 November 2015

\begin{abstract}
In order to routinely evaluate workers' internal exposure due to intake of radionuclides, a whole-body counter (WBC) at the Third Qinshan Nuclear Power Co. Ltd. (TQNPC) is used. Counting would typically occur immediately after a confirmed or suspected inhalation exposure. The counting geometry would differ as a result of the height of the individual being counted, which would result in over- or underestimated intake(s). In this study, Monte Carlo simulation was applied to evaluate the counting efficiency when performing a lung count using the WBC at the TQNPC. In order to validate the simulated efficiencies for lung counting, the WBC was benchmarked for various lung positions using a ${ }^{137} \mathrm{Cs}$ source. The results show that the simulated efficiencies are fairly consistent with the measured ones for ${ }^{137} \mathrm{Cs}$, with a relative error of $0.289 \%$. For a lung organ simulation, the discrepancy between the calibration phantom and the Chinese reference adult person $(170 \mathrm{~cm})$ was within $6 \%$ for peak energies ranging from $59.5 \mathrm{keV}$ to $2000 \mathrm{keV}$. The relative errors vary from $4.63 \%$ to $8.41 \%$ depending on the person's height and photon energy. Therefore, the simulation technique is effective and practical for lung counting, which is difficult to calibrate using a physical phantom.
\end{abstract}

Keywords: whole-body counter / Monte Carlo simulation / lung counting / counting efficiency

\section{Introduction}

A whole-body counter (WBC) is used to determine the total activity of radionuclides within a body. The counter consists of a $\gamma$-ray shielding spectrometer and the measured object is a person. Counting is typically conducted soon after a confirmed or suspected inhalation exposure. As inhaled radionuclides would remain in the lungs shortly after exposure, a lung count is usually performed. In order to obtain peak efficiencies, an anthropomorphic phantom containing known activity is required. Standardized phantoms recommended by an international organization (ICRP, 2002), which more or less meet the physical characteristics of a representative subject, are generally applied. To accurately evaluate internal contamination, a more realistic phantom is preferred. However, in practice, it is almost impossible to fabricate a realistic phantom for each case, as the individuals being counted are different one from another. Therefore, the uncertainty associated with counting efficiency remains an issue.

Monte Carlo simulations may provide an alternative method of calibration by using a numerical phantom instead of a physical one. Some papers have already demonstrated the

\footnotetext{
^ dongming-1i@126.com
}

potential and application of Monte Carlo simulation in lung counting (Kramer, 2006; Nogueira et al., 2010). The main advantage of such a method is that peak efficiencies can be obtained for various body sizes of the subjects being counted, as well as for organs or tissues where the distribution of deposited radionuclides are very inhomogeneous, which makes it impossible to mimic using a physical phantom. Recent progress in computer technology also enables more complex and realistic simulations to be performed (Ay et al., 2005; Carinou et al., 2007; Zhang, et al., 2014).

In this paper, a Monte Carlo simulation was performed to obtain peak efficiencies for lung counting at various lung positions using a whole-body counter equipped with dual detectors at the Third Qinshan Nuclear Power Co. Ltd. (TQNPC). Breustedt reported the application of Monte Carlo simulation to a whole-body counter equipped with thalliumactivated sodium iodide [ $\mathrm{NaI}(\mathrm{Tl})]$ (Breustedt et al., 2010), while Broggio utilized Monte Carlo modeling for in vivo lung monitoring of enriched uranium (Broggio et al., 2012). In this study, we calibrated the whole-body counter using Monte Carlo simulations for lung counting. The correction factors for individuals of different heights are obtained by simulation, and used to correct the measurement data from lung counting. 


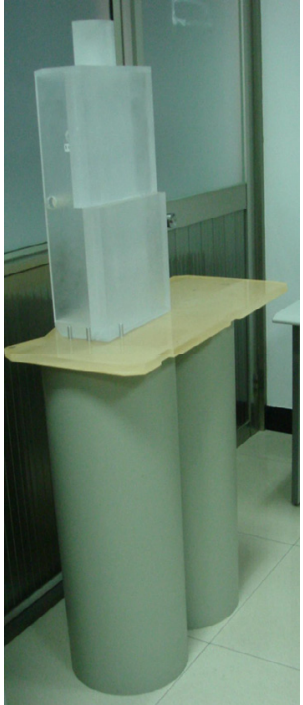

Figure 1. The 3D Picture of the Canberra Model 2257 calibration phantom.

\section{Materials and methods}

\subsection{Instrument and whole-body system}

The TQNPC uses a whole-body counter with dual $\mathrm{NaI}(\mathrm{Tl})$ detectors configured in a linear array on a common vertical axis. The counter is shielded within a room that has lowbackground steel walls and a ceiling/floor $10 \mathrm{~cm}$ thick. The counter is composed of two large sodium iodide detectors $(\mathrm{NaI}(\mathrm{Tl}))$ of $7.6 \mathrm{~cm} \times 12.7 \mathrm{~cm} \times 40.6 \mathrm{~cm}$ with a single photomultiplier tube at the end, and provides a typical Lower Limit of Detection of approximately $150 \mathrm{~Bq}(4 \mathrm{nCi})$ for ${ }^{60} \mathrm{Co}$ with a count time of one minute (Uenoa et al., 2014). The dual detector design provides a uniform or flat $( \pm 15 \%)$ response along the longitudinal axis from the thyroid of the tallest 99th percentile male to the lower gastrointestinal tract of the shortest female. In this study, the response functions of the detectors for the phantom were measured to obtain peak efficiencies, which were compared with those of the simulation results.

The calibration phantom provided by Canberra ${ }^{\circledR}$ is a simplified model without considering the size and distribution of the body organs. All of the organs (lungs, abdomen, etc.) are equivalent to a point, which would give an error for measuring the lung position for low-energy photons.

The peak analysis of the response functions was performed using commercially available software (Canberra Industries, Inc.).

\subsection{Description of phantoms}

\subsubsection{Calibration phantom}

The calibration phantom (Canberra Model 2257) contains two parts (Figure 1): the upper part and the lower part, respectively. The upper part of the calibration phantom used

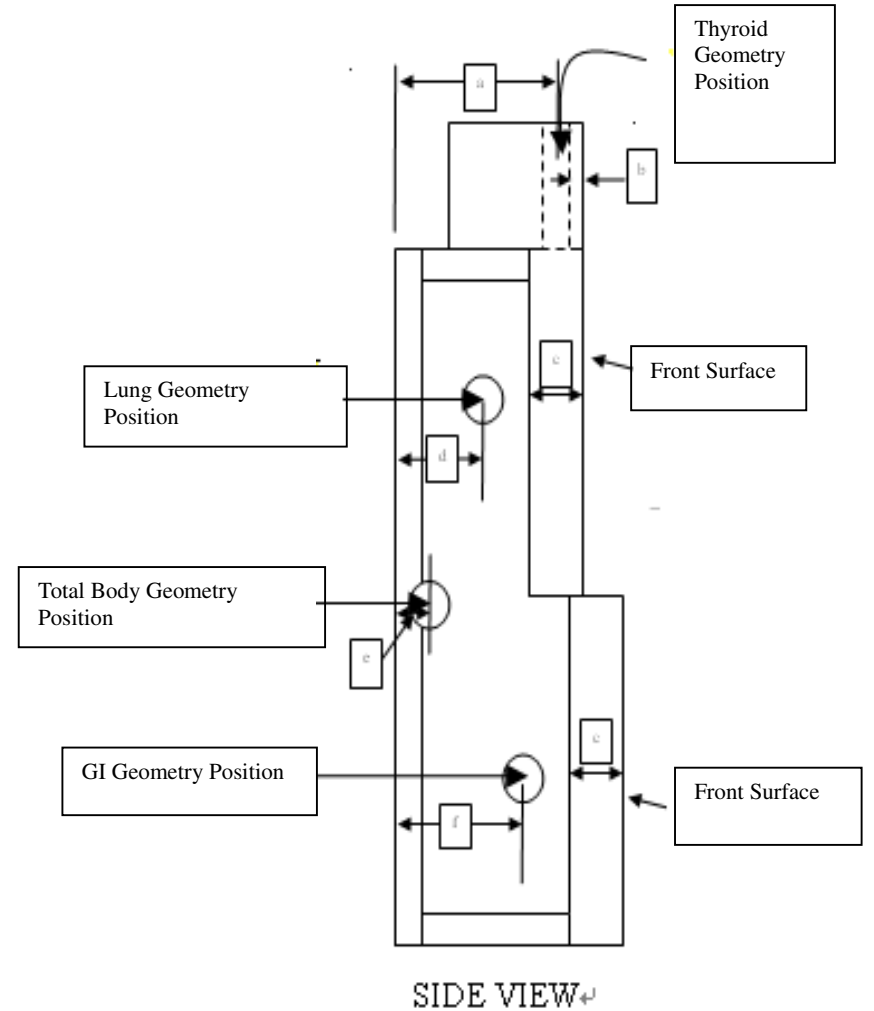

Figure 2. The upper part of calibration phantom.

in this study consists of 6 cylindrical vessels which are uniformly filled with radioactivity with soft tissue-equivalent density. The calibration radionuclides are ${ }^{241} \mathrm{Am},{ }^{109} \mathrm{Cd},{ }^{113} \mathrm{Sn}$, ${ }^{137} \mathrm{Cs},{ }^{60} \mathrm{Co}$ and ${ }^{88} \mathrm{Y}$, whose major photon peaks cover an energy range sufficient for obtaining the efficiency curve. The relative intrinsic uncertainty of the activities is $5 \%$.

The upper part of the phantom contains three sections (Figure 2), which are the neck, chest and abdomen, respectively. The chest size of the phantom is $30.5 \mathrm{~cm}$ high, $34.5 \mathrm{~cm}$ long and $18 \mathrm{~cm}$ wide. The lower part of the phantom is $30.5 \mathrm{~cm}$ high, $34.5 \mathrm{~cm}$ long and $21.5 \mathrm{~cm}$ wide. The phantom material is solid Plexiglas with the chemical formula $\mathrm{C}_{5} \mathrm{H}_{8} \mathrm{O}_{2}$ and nominal density of $1.2 \mathrm{~g} \mathrm{~cm}^{-3}$.

\subsubsection{Chinese adult person phantom}

The Chinese reference man phantom $(60 \mathrm{~kg})$ was taken as the adult male reference phantom. The geometry of this phantom is given in Table 1 . In this paper, we only simulated the chest part. The phantom material is composed of carbon $(24.2 \%)$, hydrogen $(12.2 \%)$ and oxygen $(63.6 \%)$, and the nominal density $=1.01 \mathrm{~g} \mathrm{~cm}^{-3}$.

The reference man of the Oak Ridge National Laboratory is known as the MIRD (Medical Internal Radiation Dose) Phantom. Each lung of the reference man is represented by half an ellipsoid. The section of the phantom is a similar circle (Snyder et al., 1978). References to anatomical data are given by the Chinese adult person phantoms (Wang et al., 1998). We simplified the geometry of the chest model as follows (Figure 3 ). The section of the chest model is a similar 
Table 1. Data of the Chinese adult person phantom.

\begin{tabular}{lcccccc}
\hline $\begin{array}{l}\text { Person } \\
\text { phantom }\end{array}$ & $\begin{array}{c}\text { Height } \\
(\mathbf{c m})\end{array}$ & $\begin{array}{c}\text { Weight } \\
(\mathbf{k g})\end{array}$ & $\begin{array}{c}\text { Depth of } \\
\text { chest }(\mathbf{c m})\end{array}$ & $\begin{array}{c}\text { Circle of } \\
\text { chest }(\mathbf{c m})\end{array}$ & $\begin{array}{c}\text { Depth of } \\
\text { waist }(\mathbf{c m})\end{array}$ & $\begin{array}{c}\text { Circle of } \\
\text { waist }(\mathbf{c m})\end{array}$ \\
\hline Data & 170 & 60 & 22 & 90 & 11 & 74 \\
\hline
\end{tabular}

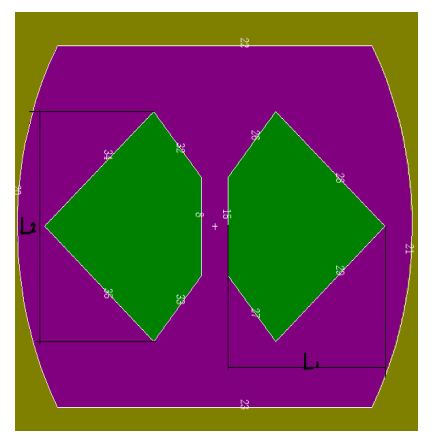

VERTICAL VIEW

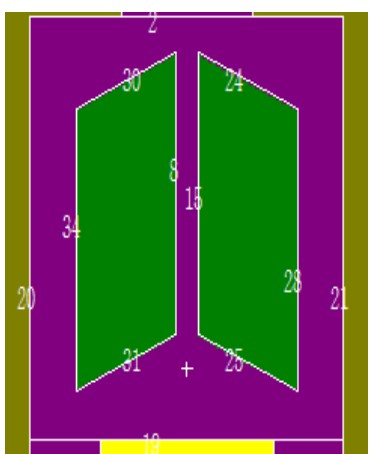

FRONT VIEW
Figure 3. The chest model of the Chinese adult person phantom.

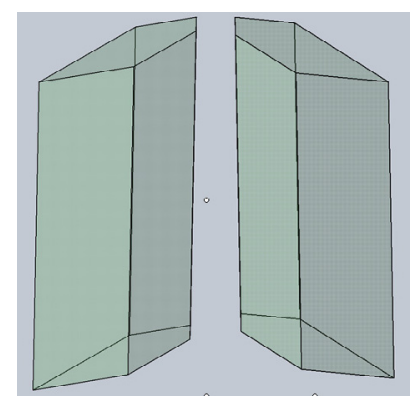

Figure 4. The 3D graph of the lung model of the Chinese adult person phantom.

rectangle, with vertical height of $30 \mathrm{~cm}$, width of $28.5 \mathrm{~cm}$ and depth of $22 \mathrm{~cm}$. We selected the section of the lung as a pentagon (Figure 4). The values of the single lung are: length of $11.5 \mathrm{~cm}$, and width of $14 \mathrm{~cm}$. The area of the section is $91 \mathrm{~cm}^{2}$, and the volume of it is $1820 \mathrm{~mL}$. The goal is to make the mathematical equations simple, thus minimizing computation time.

\subsection{Monte Carlo simulation}

Peak efficiencies for the phantoms are obtained by the Monte Carlo simulation using the MCNP4C code (Briesmeister, 2000). This code provides a general-purpose program that can simulate photon interactions with associated electron transport and production of X-rays (Peter et al., 2014) and bremsstrahlung radiation (Huang et al., 2014). It uses continuous energy data libraries over a wide range of energies for various cross-sections and scattering angles. The phantoms are seated in the air. In order to obtain statistical errors less than $1 \%$, a number of up to $1 \times 10^{8}$ histories is required.

In this study, a pulse height tally, which is provided with the MCNP4C code, was used for obtaining peak efficiencies. Peak efficiency is obtained from the probability in the energy bin and is corrected by subtracting the average probabilities
Table 2. Results of measurement and simulation for the calibration phantom.

\begin{tabular}{lccc}
\hline $\begin{array}{l}\text { Nuclide } \\
(\mathrm{MeV})\end{array}$ & $\begin{array}{c}\text { Measured efficiency } \\
\text { of calibration } \\
\text { phantom }\end{array}$ & $\begin{array}{c}\text { Simulated efficiency } \\
\text { of calibration } \\
\text { phantom }\end{array}$ & $\begin{array}{c}\text { Relative } \\
\text { error }\end{array}$ \\
\hline${ }^{137} \mathrm{Cs}(0.662)$ & $6.93 \times 10^{-3}$ & $6.91 \times 10^{-3}$ & $0.289 \%$ \\
\hline
\end{tabular}

in both sides of the energy bin as a Compton continuum. The Monte Carlo energy-bin width is set at $0.2 \mathrm{keV}$ and uniformly distributed within volume sources. A folding technique with a Gaussian distribution is not used in the simulation. Firstly, the calibration phantom efficiencies of the lung position for ${ }^{137} \mathrm{Cs}$ were simulated. The data were compared with the measurement of the same position. ${ }^{137} \mathrm{Cs}$ is a very good calibration source, because of its single gamma energy and long half-life. Secondly, calculations were performed for major peak energies of nuclides contained in the lung geometry position of the calibration phantom and the lung of the adult person phantom. The major peak energies include $0.059 \mathrm{MeV}, 0.08 \mathrm{MeV}, 0.28 \mathrm{MeV}$, $0.48 \mathrm{MeV}, 0.68 \mathrm{MeV}, 0.88 \mathrm{MeV}, 1.08 \mathrm{MeV}, 1.28 \mathrm{MeV}$, $1.48 \mathrm{MeV}, 1.68 \mathrm{MeV}, 1.8 \mathrm{MeV}$ and $2.0 \mathrm{MeV}$. Finally, calculations were performed for full energy peaks of some nuclides $\left({ }^{109} \mathrm{Cd}\right.$ and $\left.{ }^{137} \mathrm{Cs}\right)$ contained in the lung geometry position of the Chinese adult person phantom.

Each photon energy was simulated independently. The photons interacted with both the upper and lower detectors and were tallied for their full energy so that peak efficiency was obtained by the upper detector and lower detector. The total (upper and lower combined) efficiency is simply obtained by summing the efficiencies from both detectors.

\section{Results and discussions}

The efficiency of the ${ }^{137} \mathrm{Cs}$ measurement for the calibration phantom and the simulated one is shown in Table 2, together with the relative error of the measured to the simulated efficiency. The efficiency of the actual measurements and the simulated ones is in agreement within $0.3 \%$. After validation of the methodology, the MCNP code was used to calculate the efficiency of the system for different photon energies in the phantoms. As a result, the simulation for the Chinese adult person phantom is in fairly good agreement with the calibration phantom; within $6 \%$ for energies ranging from $0.059 \mathrm{MeV}$ to $2.0 \mathrm{MeV}$. Moreover, for the calibration phantom, the efficiency varies from $5.45 \times 10^{-3}$ to $10.7 \times 10^{-3}$ for energy from $0.059 \mathrm{MeV}$ to $2.0 \mathrm{MeV}$. The results are presented in Figure 5.

The results simulated for individuals of different heights from $150 \mathrm{~cm}$ to $180 \mathrm{~cm}$ are shown in Figure 6 and Table 3. The relative error between the lowest efficiency and the highest 


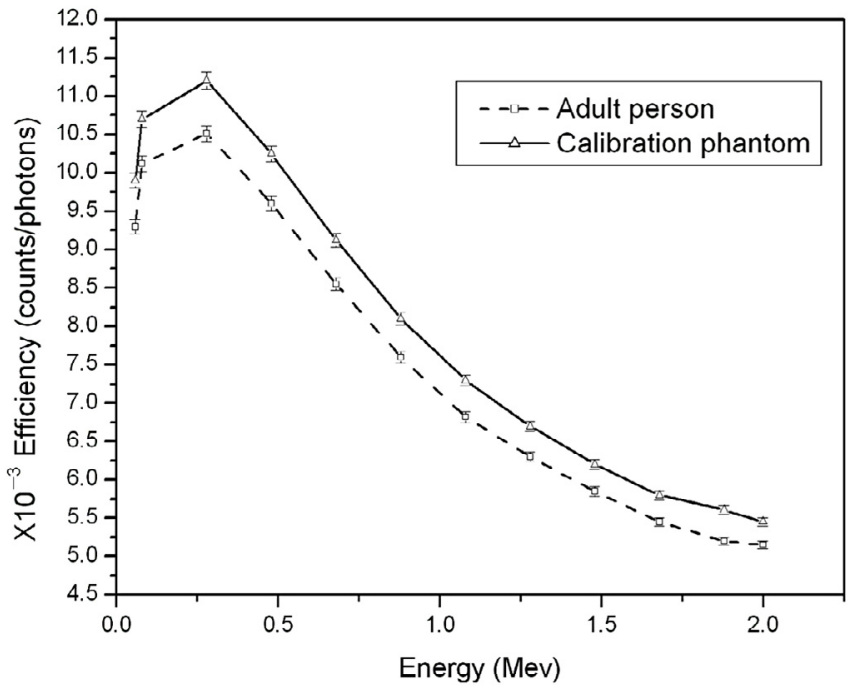

Figure 5. Efficiency curve of energy.

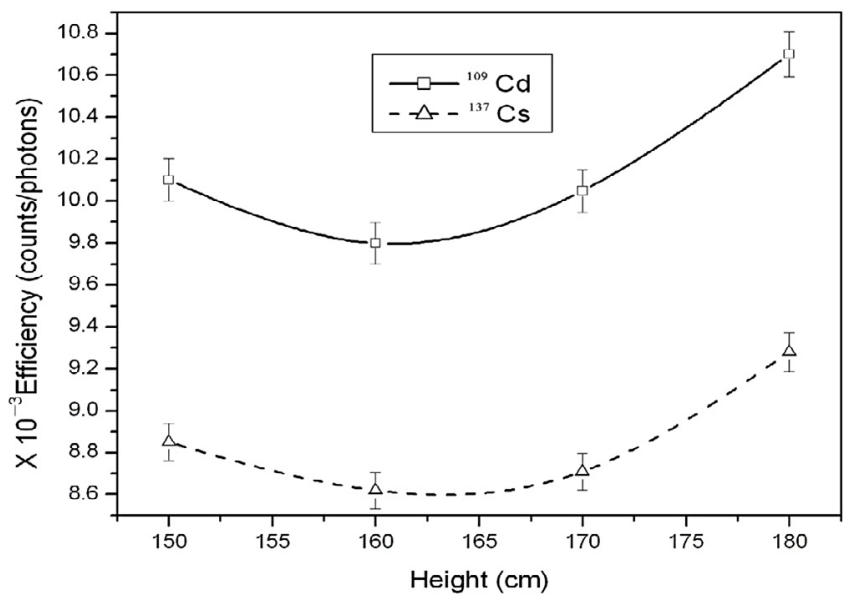

Figure 6. Efficiency curve of height.

Table 3. Results of simulation for different heights of the adult person phantom.

\begin{tabular}{lcc}
\hline Height $(\mathbf{c m})$ & ${ }^{109} \mathbf{C d}$ & ${ }^{137} \mathbf{C s}$ \\
\hline 150 & $5.61 \%$ & $4.63 \%$ \\
160 & $8.41 \%$ & $7.11 \%$ \\
170 & $6.07 \%$ & $6.14 \%$ \\
180 & 1 & 1 \\
\hline
\end{tabular}

is $8.41 \%$ and $7.110 \%$ for ${ }^{109} \mathrm{Cd}$ and ${ }^{137} \mathrm{Cs}$, respectively. This means that when a person is measured, his body size is different from the Reference Man calibration phantom, and the error introduced into the activity estimate is less than a maximum value of about $8.5 \%$ and $7.5 \%$ for ${ }^{109} \mathrm{Cd}$ and ${ }^{137} \mathrm{Cs}$, respectively. The highest uncertainty value corresponds to the lowest energy gamma emitter, ${ }^{109} \mathrm{Cd}$.

The deviation levels observed in some positions are largely due to the intrinsic uncertainties arising from several factors, such as: i) In order to evaluate peak efficiencies for the phantom of the WBC, it is necessary to build a proper model. In this simulation the entire system of the WBC including the shielding is modeled as accurately as possible. However, it is difficult to determine the detailed structure of each part of the detectors (Nogueira et al., 2010) and the lungs. ii) The density of the parts of the Canberra calibration phantom has the same value, which would give uncertainties between the calibration phantom and body phantom. iii) Different fractions of photons with different energies are absorbed in the human body, and this would affect the measurement uncertainty of the WBC. Higher deviations are expected in the lower-energy regions, where the models used for particle transport are less accurate (Joana et al., 2012)). iv) The uncertainty of standard sources, and the minimum detection level of the instrument and measurement time could also affect measurement uncertainty. v) Other parameters, such as the weight and chest, would be correctly taken into account with different heights of the phantoms, while in our simulations, the same parameters were used, which would affect measurement uncertainty as well.

\section{Conclusions}

This study demonstrated the application of Monte Carlo simulation. In this particular case, the MCNP code is a powerful tool for the estimation of counting efficiency using the WBC. The agreement between the measured and simulated efficiencies is good, within $0.289 \%$, for the measurement of ${ }^{137} \mathrm{Cs}$ in the lung. The discrepancy between the calibration phantom and the Chinese adult person phantom $(170 \mathrm{~cm})$ is within $6 \%$ for peak energies ranging from $59.5 \mathrm{keV}$ to $2000 \mathrm{keV}$. The relative errors vary from $4.63 \%$ to $8.41 \%$ depending on the person's height and photon energy. This work contributes to demonstrating the great effectiveness of using computational tools for understanding the calibration of radiation detection systems used for in vivo monitoring.

Acknowledgements. This project was financially supported by the National Natural Science Fund Committee and the Civil Aviation Administration of China Jointly Funded Project (U1233116), the Third Qinshan Nuclear Power Co. Ltd. (TQNPC) and the Doctoral Research Fund of Xian Shiyou University (2014BS42). We are grateful for the contribution and support of the staff at the Department of Health Physics of the TQNPC. We acknowledge the kind help of Professor Shang of the Second Artillery Engineering College of China, and Dave Groff (Senior Health Physicist of Canberra Industries, Inc.) who provided us with their data on calibration phantoms.

\section{References}

Ay M.R. et al. (2005) MCNP4C-based Monte Carlo simulator for fan and cone-beam X-ray CT: development and experimental validation, Biomedizinische Technik 50, 360-361.

Breustedt B. et al. (2010) Monte Carlo calibration of whole-body counters with $\mathrm{NaI}(\mathrm{Tl})$ detectors in stretcher geometry, Radiat. Prot. Dosim. 139 (4), 510-518.

Broggio D. et al. (2012) Monte Carlo modelling for the in vivo lung monitoring of enriched uranium: Results of an international comparison, Radiat. Meas. 47, 492-500.

Briesmeister F. (2000) MCNP-A general Monte Carlo N-particle transport code version 4C, Los Alamos National Laboratory Report LA-13709-M. 
Carinou E. et al. (2007) The calculation of a size correction factor for a whole-body counter, Nucl. Instrum. Meth. Phys. Res. A 580,197-200.

ICRP Publication 89 (2002) Basic Anatomical and Physiological Data for Use in Radiological Protection: Reference Values, Ann. ICRP 32 (3-4).

Joana B. et al. (2012) Monte Carlo Simulation of the Movement and Detection Efficiency of a Whole-body Counting System Using a BOMAB Phantom, Radiat. Prot. Dosim. 148 (4), 403-413.

Kramer G.H. (2006) The sliced BOMAB phantom: a new variant for intercomparison, Health Phys. 90, 161-166.

Huang J.-W. et al. (2014) Efficiency calibration for a NaI scintillation detector based on Monte Carlo process and preliminary measurements of bremsstrahlung, Acta Physica Sinica 63, 180702.

Nogueira P. et al. (2010) Monte Carlo simulation of the full energy peak efficiency of a WBC, Appl. Radiat. Isotopes 68, 184-189.
Peter S. et al. (2014) Combining Monte Carlo methods with coherent wave optics for the simulation of phase-sensitive X-ray imaging, J. Synchrotron Radiat. 21, 613-622.

Snyder W.S. et al. (1978) Estimates of absorbed fractions for monoenergetic photon source uniformly distributed in various organs of a heterogeneous phantom. The Society of Nuclear Medicine; Medical Internal Radiation Dose Committee (MIRD), New York. Pamphlet No. 5.

Uenoa K. et al. (2014) On-line ${ }^{60} \mathrm{Co}$ monitor for reactor recirculation system piping in primary containment vessel during reactor operation, Nucl. Eng. Design 278, 352-359.

Wang J. et al. (1998) Data of Anatomical Physiological and Metabolizing Characteristics for Chinese Referrence Man. China Atomic Energy Press, Beijing (in Chinese).

Zhang J. et al. (2014) Monte Carlo Simulation and Analysis of $\gamma$ Rays for the Important Elements of Lunar Surface, Nucl. Phys. Rev. 31, 112-117.

Cite this article as: L. Dongming, J. Shuhai, L. Houwen. Monte Carlo simulation of lung counting efficiency using a whole-body counter at a nuclear power plant. Radioprotection 51(1), 59-63 (2016). 